\title{
ORIGINAL
}

\section{Effect of hormone replacement therapy on total serum anti-oxidant potential and oxidized LDL/ß2-glycoprotein I complexes in postmenopausal women}

\author{
Maryam Darabi ${ }^{1)}$, Mohsen Ani ${ }^{1)}$, Ahmad Movahedian ${ }^{1), 2)}$, Elahe Zarean ${ }^{3)}$, Mojtaba Panjehpour ${ }^{1)}$ and \\ Mohammed Rabbani ${ }^{4}$ \\ ${ }^{1)}$ Department of Clinical Biochemistry, School of Pharmacy and Pharmaceutical Sciences, Isfahan University of Medical Sciences, \\ Isfahan, Iran \\ ${ }^{2)}$ Isfahan Pharmaceutical Sciences Research Center, Isfahan University of Medical Sciences, Isfahan, Iran \\ 3) Department of Gynecology and Obstetrics, School of Medicine, Isfahan University of Medical Science, Isfahan, Iran \\ ${ }^{4)}$ Department of Biotechnology, School of Pharmacy and Pharmaceutical Sciences, Isfahan University of Medical Sciences, \\ Isfahan, Iran
}

\begin{abstract}
Oxidative stress and consequent oxidized lipoprotein production is thought to play a central role in both the initiation and progression of atherosclerosis. Oxidized low-density lipoprotein (oxLDL)/ $\beta 2$-glycoprotein I ( $\beta 2 \mathrm{GPI})$ complexes are etiologically important in the development of atherosclerosis. The aim of the present study was to investigate whether long-term treatment with conventional hormone replacement therapy (HRT) in postmenopausal women could affect total serum antioxidant capacity (TAC) and serum levels of oxLDL/ $32 \mathrm{GPI}$ complexes. A total of 60 normolipidemic postmenopausal women treated with oral estrogen together with progestin therapy for 3 months were selected. TAC and serum levels of oxLDL/ $\beta 2$ GPI complexes were measured at the beginning and end of the HRT. HRT led to a significant increase in TAC $(15 \%, P=0.02)$ and a minor but statistically nonsignificant decrease of oxLDL/ $\beta 2$ GPI complexes $(3 \%$, $P=0.30$ ) when compared with the baseline control levels. There was also no significant association between TAC and oxLDL/ $\beta 2$ GPI complexes changes related to HRT. This study indicates that, HRT in postmenopausal women leads to an increase in TAC without an equivalent change in serum levels of oxLDL/ $\beta 2$ GPI complexes. It is concluded that beneficial effects of HRT could be explained, at least in part, by improving antioxidant status, but may not be directly associated with a change in oxidized lipoprotein production.
\end{abstract}

Key words: Postmenopause, Antioxidant status, HRT, oxLDL/32GPI complexes, TAC

THE IMPORTANCE of oxidative stress has been indicated by increased plasma and arterial lipid peroxide and altered antioxidant status in atherosclerotic patients [1]. The uptake of oxidized LDL (oxLDL) by monocytes located in the arteries may lead to their transformation into macrophages, residential macrophages and finally foam cells [2]. OxLDL interacts with $\beta$-2-glycoprotein-I ( $\beta 2 \mathrm{GPI})$, forming circulating oxLDL/ß2GPI complexes that are etiologically important in the formation of foam cells, an early stage of

Received Aug. 9, 2010; Accepted Sep. 25, 2010 as K10E-239 Released online in J-STAGE as advance publication Oct.22, 2010

Correspondence to: Ahmad Movahedian, Isfahan Pharmaceutical Sciences Research Center, School of Pharmacy and Pharmaceutical Sciences, Isfahan University of Medical Sciences, Isfahan, Iran. E-mail: movahedian@pharm.mui.ac.ir atherosclerosis. Recent studies have shown that the measurement of oxLDL/ $\beta 2$ GPI complexes in patients with cardiovascular diseases provides a novel marker for those patients prone to or at risk for coronary heart disease [3].

Numerous observational studies indicate that postmenopausal hormone replacement therapy (HRT) is associated with lower risk for coronary heart disease $[4,5]$. Several hypotheses regarding the atheroprotective effect of HRT have been proposed. Some studies have linked the development of protective effects to an improved antioxidant status [6,7]. The mechanism responsible for HRT-associated oxidative stress is not fully elucidated. Estrogen has been reported to have potent antioxidant properties, which may exert their influence through reducing oxidized lipids [8]. 
In this study, the effects of HRT on serum levels of oxLDL/ $\beta 2$ GPI complexes were investigated in nonobese normolipidemic postmenopausal subjects. We also measured the total serum antioxidant capacity (TAC) as ferric reducing ability of plasma (FRAP) and related these to HRT and oxLDL/B2GPI complexes level. We hypothesized that HRT would relate positively with antioxidant status.

\section{Materials and Methods}

\section{Subjects}

Sixty nonobese normolipidemic postmenopausal women $\left(54.7 \pm 4.6\right.$ years of age, BMI $\left.25.2 \pm 1.6 \mathrm{~kg} / \mathrm{m}^{2}\right)$ were selected through personal contacts of more than 400 volunteers who were admitted to two clinical centers at the Isfahan University of Medical Sciences. The mean age at menopause in the population was $46.5 \pm 3.7$ years. Details of the study design and sampling have been reported earlier [9]. Briefly, Women were considered postmenopausal if they had no menstrual periods for at least 12 months and serum follicle-stimulating hormone (FSH) of $>35 \mathrm{IU} / \mathrm{L}$ without other obvious pathological or physiological cause. Exclusion criteria were age $\geq 65$ years, BMI $\geq 30 \mathrm{~kg} / \mathrm{m}^{2}$, a previous hospital admission related to cardiovascular disease, and a previous diagnosis of angina, hypercholesterolemia, or diabetes. In addition, none of them had smoking habits or had taken medications or vitamin supplements in the 4 months before the study. During the study period the subjects maintained their regular physical lifestyle and activity. The subjects were asked to record any consumption of drugs not included in the experimental design.

Study participants were assigned to a 90-day experimental period. During the experimental period all subjects received oral HRT with estrogen plus progestagen $(0.625 \mathrm{mg}$ of conjugated equine estrogen plus $2.5 \mathrm{mg}$ of medroxyprogesterone acetate per day; Iran Hormone Co., Iran). The study protocol was approved by the institutional review board and the ethic committee of Isfahan University of Medical Sciences, and all patients gave written informed consent. Evaluation of treatment compliance was ascertained by weekly interview as well as by pill count.

\section{Blood sampling and analysis}

Fasting blood samples were taken at the beginning of the study and at the end of hormone therapy. Serum aliquots were obtained by centrifugation. Samples were deep frozen for later analysis. Serum total cholesterol, triglyceride (TG), HDL-C and LDL-C were determined by using standard enzymatic procedures [10] on a BT 3000 autoanalyzer (Biotecnica Institute, IT). TAC was determined by FRAP method, which is based on the reduction of a colorless ferric tripyridyltriazine complex to a blue ferrous complex by the antioxidants in the plasma. The change in absorbance at $593 \mathrm{~nm}$ is directly related to the total reducing power of electron donating antioxidants present in the plasma [11]. The serum oxLDL/ $\beta 2$ GPI complexes concentration was determined using ELISA (Cayman Chemical, Ann Arbor, MI). The overall coefficients of variation for these assays were between 1.9 and 5.2\%. All analyses were run in a blinded fashion.

\section{Data analyses}

Variables were analysed for distribution and a logarithmic transformation was applied to the values of TG/HDL-C ratio to normalize distribution of the data. $P$-value $<0.05$ was considered statistically significant. All analyses were carried out using SPSS for windows version 11.0 (SPSS Inc., Chicago, IL).

\section{Results}

Compliance to the experimental intervention was good according to data records. The HRT led to a significant reduction in LDL-C $(P=0.02)$ as compared with the baseline (Table 1). Hormone therapy was associated with significantly higher concentrations of HDL-C $(P=0.001)$. Also, beneficial modifications expressed as a non-significant decrease in the log TG/HDL-C ratio ( $P=0.061)$ were observed at the third month of treatment with respect to basal values.

Fig. 1 show parameters related to the oxidant-antioxidant status before hormone therapy and after 90 days of therapy. Analysis of TAC by FRAP method found a significantly higher antioxidant capacity $(15 \%$, $P=0.024$ ) mean value at the third month than at the baseline. However, there was no statistically significant change in the oxLDL/ $\beta 2$ GPI complexes level $(3 \%, P=0.30)$ in response to HRT. The ratio of oxLDL/ $\beta 2$ GPI to HDL-C which expresses lipoprotein oxidative status [12], were calculated in each case. The oxLDL/B2GPI to HDL-C ratio was significantly lower in samples obtained after HRT ( $P=0.018$, Fig. 1). Our data showed no significant correlation between TAC and oxLDL/B2GPI complexes changes related to HRT. 
Table 1 Effects of hormone replacement therapy (HRT) on serum lipid and lipoprotein levels ${ }^{1}$

\begin{tabular}{lccc}
\hline & Baseline & HRT, $3^{\text {rd }}$ month & $P$ \\
\hline Cholesterol (mg/dL) & $202 \pm 41$ & $200 \pm 35$ & 0.52 \\
Triglyceride (mg/dL) & $148 \pm 51$ & $150 \pm 53$ & 0.59 \\
HDL cholesterol (mg/dL) & $45 \pm 11$ & $50 \pm 11$ & 0.001 \\
LDL cholesterol (mg/dL) & $124 \pm 27$ & $116 \pm 25$ & 0.02 \\
$\log$ Triglyceride/HDL cholesterol & $0.51 \pm 0.19$ & $0.46 \pm 0.18$ & 0.061 \\
\hline
\end{tabular}

${ }^{1}$ Values are expressed as means $\pm \mathrm{SD}, P<0.05$ (paired $t$-test).
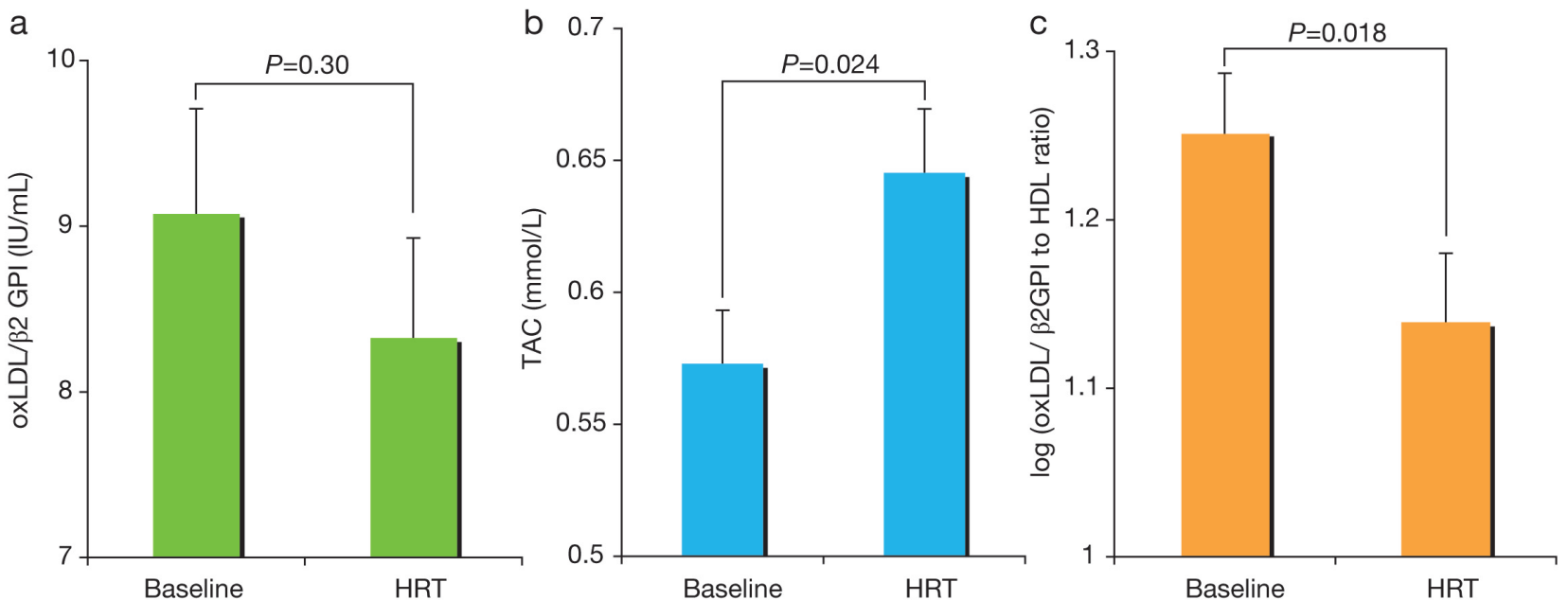

Fig. 1 Effects of hormone replacement therapy (HRT) on oxLDL/ $/ 2$ GPI (a), total antioxidant capacity (TAC) (b), oxLDL/ $\beta 2$ GPI to HDL-C ratio (c) in the studied subjects. Values are means \pm SE. $P<0.05$ (paired sample $t$-test).

OxLDL/B2GPI complexes level was correlated with total cholesterol $(r=0.31, P=0.01)$ and LDL-C $(r=0.28$, $P=0.03$ ) both before and after HRT. No similar association was observed for TAC and other lipid parameters.

\section{Discussion}

Postmenopausal hormone therapy has been generally recognized as a component of preventive health care for elderly women, with many benefits including improvement in climacteric symptoms and prevention of osteoporosis and cardiovascular disease [13, 14]. Controversies still exist regarding the beneficial protecting effects of HRT. Several clinical trials demonstrated that HRT may increase the risk of cardiovascular complication. The results of these clinical trials are in contrast to those of observational studies which suggested a protective role of hormone therapy [4]. Postmenopausal hormone therapy may exert its antiatherogenic effect through their antioxidant properties, preventing oxidative modification of LDL, as well as foam-cell formation and atherogenesis [15]. Recently, interest has focused on novel plasmatic biomarkers of lipid peroxidation such as levels of oxLDL/32GPI complexes. For the first time, we examined whether conventional HRT supplementation in postmenopausal women could affect their oxLDL/ $\beta 2$ GPI complexes.

Adherence to the HRT was supported by the quality of the treatment records. The effects on serum lipid composition found after the HRT agree with previous studies [16] and has been discussed earlier by us [9].

Total antioxidative capacity of serum is related to nonenzymatic and enzymatic systems. TAC measurement is considered to be the most appropriate way to assess the performance of the entire antioxidant system. The effects of short- and long-term HRT on plasma antioxidant status has been demonstrated in a wide variety of studies. Hernandez et al. [17] have shown that estrogen intake is associated with an increase in plasma levels of anti-oxidants of ovariectomized rats. In accor- 
dance, determination of the TAC by FRAP assay in our study showed a significant increase in antioxidant status of plasma by HRT. The 3-month exposure time was considered long enough because comparable clinical studies with hormone therapy demonstrate significant changes in serum lipids and antioxidative enzyme activity $[18,19]$ or even during the menstrual cycle [20].

It has been reported that estradiol combined with intrauterine levonorgestrel decreased LDL oxidation in vivo [21]. Accordingly, lower lipid peroxidation were found in postmenopausal women with both the estrogen alone and combined with progestin oral HRT when compared to the control group [22], suggesting that HRT is beneficial in the protection against oxidative stress. In our study we observed that hormone therapy in postmenopausal groups caused an increase in TAC and an increase in HDL, but we did not observe a change in oxLDL/ $\beta 2$ GPI complexes. There may be a complex interaction between different progestins and routes of HRT administration on metabolic and oxidative markers [22-24]. Therefore, the magnitude and type of effect according to the progesterone content and route of administration may vary between studies. In line with our study, it has been shown that the lipoprotein susceptibility to oxidation, assessed by ex vivo analyses, was not affected by oral combined HRT with estradiol and dydrogesteron [25]. Controversially, it has been shown that oral conjugated equine estrogen induced increase in plasma TG concentration can reduce the size of LDL particles, which are more susceptible to oxidation [24]. Therefore, a possible increase in susceptibility of LDL to oxidation during HRT in the present study may be an explanation for there being no change in oxLDL/B2GPI complexes despite a significant improvement in serum antioxidant potential. OxLDL, not native LDL, binds $\beta 2$ GPI forming stable complexes. It has been suggested that oxLDL/B2GPI complexes are formed in the arterial wall where they are either internalized by macrophages to form foam cells and/or released back into the circulation [26]. It is possible that the interaction between $\beta 2$ GPI and oxLDL reduces the inflammatory properties of oxLDL while promoting its clearance from circulation [27]. HRT may influence the formation and clearance of oxLDL/ $32 \mathrm{GPI}$ complexes partly through nonantioxidative processes by changing $\beta 2 \mathrm{GPI}$ expression level and/or macrophage scavenging activity. In support of this hypothesis, it has been shown that $\beta 2$ GPI is down regulated during inflammation [28]. Stable oxLDL/B2GPI complexes exert an indirect but signifi- cant role in atherosclerosis, which is still being elucidated. We found that oxLDL/B2GPI complexes correlated with total cholesterol and LDL-C, possibly reflecting the known positive correlation of cholesterol with atherosclerosis. Future studies examining multiple oxidative stress markers simultaneously will be required to attain a better understanding of the potential role of HRT in oxidative stress.

Because oxidative stress results primarily from an imbalance between oxidant and antioxidants, a combination of markers reflecting these two components appears to be clinically useful. The antioxidant properties of HDL have been well studied and the ratio of oxLDL/B2GPI complexes to HDL-C probably reflects better oxidative stress. Earlier reports suggest that, this ratio imbalance could be a useful marker for atherosclerosis in diabetic patients $[12,29]$. In the current study, we have found a significant decrease in the oxLDL/ $\beta 2$ GPI complexes to HDL-C ratio after HRT. These findings further support the findings of a less oxidative stress by the setting of HRT described here. The role of HRT as antioxidant in vivo is still not satisfactory resolved [30]. The effect of combined estrogen/ progestin therapy, which is now the preferred treatment for women with an intact uterus, has been investigated in our study. Future studies may focus on the impact of age and the differential effects of estrogen/progestin and estrogen only therapy.

Our findings showed that 3 months of HRT increased the plasma total antioxidant capacity without having a significant effect on oxLDL/ $\beta 2$ GPI complexes. This might be explained by either different mechanism involved in oxLDL/ $\beta 2$ GPI complexes formation compared with oxLDL formation, or by an interfering effect of HRT on the susceptibility of LDL to oxidation. In conclusion, we propose that beneficial effects of HRT could be explained, at least in part, by improving antioxidant status, but may not be directly associated with altering the oxidized lipoprotein production.

\section{Acknowledgment}

This study was supported by a grant from the Isfahan University of Medical Sciences.

\section{Conflict of interest}

The authors declare that they have no conflict of interest. 


\section{References}

1. Meisinger C, Baumert J, Khuseyinova N, Loewel H, Koenig W (2005) Plasma oxidized low-density lipoprotein, a strong predictor for acute coronary heart disease events in apparently healthy, middle-aged men from the general population. Circulation 112: 651-657.

2. Jessup W, Kritharides L (2000) Metabolism of oxidized LDL by macrophages. Curr Opin Lipidol 11: 473-481.

3. Greco TP, Conti-Kelly A M, Anthony J R, Greco T, Jr., Doyle R, Boisen M, Kojima K, Matsuura E, Lopez L R (2010) Oxidized-LDL/beta(2)-glycoprotein I complexes are associated with disease severity and increased risk for adverse outcomes in patients with acute coronary syndromes. Am J Clin Pathol 133: 737-743.

4. Barrett-Connor EGrady D (2003) Hormone replacement therapy, heart disease, and other considerations. Annu Rev Public Health 19: 55-72.

5. Grodstein F, Stampfer M J, Manson J E, Colditz G A, Willett W C, Rosner B, Speizer F E, Hennekens C H (1996) Postmenopausal estrogen and progestin use and the risk of cardiovascular disease. $N$ Engl J Med 335: 453-461.

6. The Writing Group for the PEPI Trial, Miller V T, LaRosa J, Barnabei V, Kessler C, Levin G, Smith-Roth A, Griffin M, Stoy D B, Bush T, Zacur H, Foster D and others (1995) Effects of estrogen or estrogen/ progestin regimens on heart disease risk factors in postmenopausal women: The postmenopausal estrogen/progestin interventions (PEPI) trial. JAMA 273: 199-208.

7. Espeland MA, Marcovina S M, Miller V, Wood P D, Wasilauskas C, Sherwin R, Schrott H, Bush T L (1998) Effect of Postmenopausal hormone therapy on lipoprotein(a) concentration. Circulation 97: 979-986.

8. Lamon-Fava S, Postfai B, Diffenderfer M, DeLuca C, O'Connor J, Jr., Welty F K, Dolnikowski G G, Barrett P H, Schaefer E J (2006) Role of the estrogen and progestin in hormonal replacement therapy on apolipoprotein a-i kinetics in postmenopausal women. Arterioscler Thromb Vasc Biol 26: 385-391.

9. Darabi M, Rabbani M, Ani M, Zarean E, Panjehpour M, and Movahedian A (2010) Increased leukocyte ABCA1 gene expression in postmenopausal women on hormone replacement therapy. Gynecol Endocrinol [In press] early online: doi 10.3109/09513590.2010.507826.

10. Rifai N, Warnick G (2006) Lipids, lipoproteins, apolipoproteins, and other cardiovascular risk factors. In: Burtis C, Ashwood E, Bruns D (26ed) Tietz Textbook of Clinical Chemistry. Elsevier Saunders, Philadelphia: 903-968.

11. Benzie IF, Strain J J (1996) The ferric reducing ability of plasma (FRAP) as a measure of "antioxidant power": the FRAP assay. Anal Biochem 239: 70-76.

12. Girona J, Manzanares J M, Marimon F, Cabre A, Heras M, Guardiola M, Ribalta J, Masana L (2008) Oxidized to non-oxidized lipoprotein ratios are associated with arteriosclerosis and the metabolic syndrome in diabetic patients. Nutr Metab Cardiovasc Dis 18: 380-387.

13. Gass MLS, Bassuk S S, Manson J E (2009) Reassessing benefits and risks of hormone therapy. Am J Lifestyle Med 3: 29-43.

14. Stampfer MJColditz G A (1991) Estrogen replacement therapy and coronary heart disease: A quantitative assessment of the epidemiologic evidence. Prev Med 20: 47-63.

15. Yla-Herttuala S (1996) Expression of lipoprotein receptors and related molecules in atherosclerotic lesions. Curr Opin Lipidol 7: 292-297.

16. Rijpkema AHM, van der Sanden A A, Ruijs A H C (1990) Effects of post-menopausal oestrogen-progestogen replacement therapy on serum lipids and lipoproteins: a review. Maturitas 12: 259-285.

17. Hernandez I, Delgado J L, Diaz J, Quesada T, Teruel M J, Llanos M C, Carbonell L F (2000) 17beta-estradiol prevents oxidative stress and decreases blood pressure in ovariectomized rats. Am J Physiol Regul Integr Comp Physiol 279: R1599-R1605.

18. Massafra C (1997) Effects of estradiol and medroxyprogesterone-acetate treatment on erythrocyte antioxidant enzyme activities and malondialdehyde plasma levels in amenorrhoic women. J Clin Endocrinol Metab 82: 173-175.

19. Park JS, Jung H H, Yang W S, Kim S B, Min W K, Chi H S (2000) Effects of hormonal replacement therapy on lipid and haemostatic factors in post-menopausal ESRD patients. Nephrol Dial Transplant 15: 1835-1840.

20. Massafra C, De F C, Gioia D, Buonocore G (1998) Variations in erythrocyte antioxidant glutathione peroxidase activity during the menstrual cycle. Clin Endocrinol (Oxf) 49: 63-67.

21. Ahotupa M, Rauramo I, Vasankari T J, Skouby S O, Hakonen T (2004) Estrogen replacement therapy in combination with continuous intrauterine progestin administration reduces the amount of circulating oxidized LDL in postmenopausal women: dependence on the dose of progestin. Ann Med 36: 278-284.

22. Ozden S, Dildar K, Kadir Y H, Gulizar K (2001) The effects of hormone replacement therapy on lipid peroxidation and antioxidant status. Maturitas 38: 165-170.

23. Araujo DA, Farias M L, Andrade A T (2002) Effects of transdermal and oral estrogen replacement on lipids and glucose metabolism in postmenopausal women with type 2 diabetes mellitus. Climacteric 5: 286-292.

24. Wakatsuki A, Okatani Y, Ikenoue N, Fukaya T (2002) Different effects of oral conjugated equine estrogen and transdermal estrogen replacement therapy on size and oxidative susceptibility of low-density lipoprotein particles in postmenopausal women. Circulation 106: 1771- 
1776.

25. Bureau I, Laporte F, Favier M, Faure H, Fields M, Favier A E, Roussel A M (2002) No antioxidant effect of combined HRT on LDL oxidizability and oxidative stress biomarkers in treated post-menopausal women. $J$ Am Coll Nutr 21: 333-338.

26. Kobayashi K, Matsuura E, Liu Q, Furukawa J, Kaihara K, Inagaki J, Atsumi T, Sakairi N, Yasuda T, Voelker D R, Koike T (2001) A specific ligand for beta(2)-glycoprotein I mediates autoantibody-dependent uptake of oxidized low density lipoprotein by macrophages. $J$ Lipid Res 42: 697-709.

27. Lopez LR, Kobayashi K, Matsunami Y, Matsuura E (2009) Immunogenic oxidized low-density lipoprotein/ beta2-glycoprotein I complexes in the diagnostic management of atherosclerosis. Clin Rev Allergy Immunol
37: 12-19.

28. Sellar GC, Keane J, Mehdi H, Peeples M E, Browne N, Whitehead A S (1993) Characterization and acute phase modulation of canine apolipoprotein $\mathrm{H}$ (beta 2-glycoprotein I). Biochem Biophys Res Commun 191: 12881293.

29. Johnston N, Jernberg T, Lagerqvist B, Siegbahn A, Wallentin L (2006) Improved identification of patients with coronary artery disease by the use of new lipid and lipoprotein biomarkers. Am J Cardiol 97: 640-645.

30. Santanam N, Shern-Brewer R, McClatchey R, Castellano P Z, Murphy A A, Voelkel S, Parthasarathy S (1998) Estradiol as an antioxidant: incompatible with its physiological concentrations and function. $J$ Lipid Res 39: 2111-2118. 